\title{
Recent Advancements in CAD/CAM Same-Day Dentistry in Practice and Education
}

\author{
Sompop Bencharit ${ }^{1,2, *}$, Wendy A. Clark, ${ }^{3}$ Lisa O. Stoner, ${ }^{3}$ Gautam Chiang, ${ }^{1}$ and Taiseer A. Sulaiman ${ }^{3}$ \\ 1 Department of Oral and Craniofacial Molecular Biology, Philips Institute for Oral Health Research, School \\ of Dentistry, Virginia Commonwealth University, Richmond, VA, 23298, USA; sbencharit@vcu.edu (S.B.), \\ chiangg@vcu.edu (G.C.) \\ 2 Department of Biomedical Engineering, College of Engineering, Virginia Commonwealth University, \\ Richmond, VA, 23220, USA; sbencharit@vcu.edu (S.B.) \\ 3 Division of Comprehensive Oral Health, Adams School of Dentistry, University of North Carolina at Chapel \\ Hill, NC, 23298, USA; waclark@unc.edu (W.A.C.), lisa stoner@unc.edu (L.A.S.) , taiseer sulaiman@unc.edu \\ (T.A.S.) \\ * Correspondence: sbencharit@vcu.edu
}

\begin{abstract}
Digital dentistry, including CAD/CAM dentistry, is perhaps the most disruptive innovation in dentistry to date. The rapid development of digital dentistry technologies over the past several decades has enabled clinicians to improve patient care by significantly reducing the time necessary for the restorative phase of treatment. Advancements in intra-oral scanning and computer-aided design and manufacturing (CAD/CAM), in conjunction with new dental CAD/CAM ceramic materials, have simplified patient care and made same-day dentistry a reality. This review aims to present the most recent advancements described in current literature as well as to document the successful implementation of digital dentistry into a predoctoral program. The overall process of CAD/CAM same-day dentistry and the accompanying advancements in software and materials were presented and discussed. Implementation of technology and personnel requirements were reviewed. CAD/CAM dentistry has been influential in shaping and improving dental practice and education, and this influence will only continue with time.
\end{abstract}

Keywords: CAD/CAM; dental ceramics; dental technology; digital dentistry; dental education; dental innovation; disruptive technology

\section{Introduction}

\subsection{A Brief History of CAD/CAM Dentistry}

Francois Duret's presentation of the "Sopha System" concept--which later became the precursor to modern computer-aided design and computer-aided manufacturing (CAD/CAM) dentistry--in 1971 is often considered the birth of digital dentistry. ${ }^{1}$ Because the concept of CAD/CAM dentistry in the earlier days was far ahead of contemporary technology, the pioneer vision would not become a reality until the last 2 decades. In 1977, John Young and Bruce Altschuler developed an intraoral grid-surface mapping system that would become the basis of intraoral scanning technology. ${ }^{2}$ Digital dental technology has evolved alongside other concurrently developing technologies. For instance, in 1984, Apple Inc introduced its first Macintosh computer. ${ }^{3}$ In 1985, the CEREC system, the first commercially available CAD/CAM dentistry system, was developed by Mormann and Brandestini. ${ }^{4,5}$ In parallel with the pioneering developments in digital dentistry, the CAD/CAM industry was also evolving rapidly. In 1987, Chuck Hull introduced the first 3D printer using stereolithography (SLA), the SLA-1, and founded a 3D printing company, 3D Systems Inc. ${ }^{6}$ During the 1990s, a competitive surge of development took hold, however, significant developments have emerged most rapidly in the last 20 years. In 2000, the 3Shape company was founded as a software developer for dental laboratories. ${ }^{7}$ Nowadays, over half of all dental laboratories around the world utilize 
3Shape prosthetic design software. In terms of intraoral scanning, many companies have developed their own intraoral scanners and have established their presences in the market. iTero intraoral scanners, for example, were introduced in 2006 by Cadent and later acquired by Align Technology. iTero intraoral scanners, which were initially developed for fixed prosthodontics, have since been improved to expand applications to orthodontics. ${ }^{8}$ Materials for CAD/CAM dentistry have also evolved rapidly, especially over the past two decades. For instance, Ivoclar Vivadent introduced lithium disilicate in 2006, which is perhaps one of the most common materials currently used in CAD/CAM same-day dentistry. The development of zirconia restorative material has also competitively evolved, particularly in posterior and implant-supported restorations.

In 2015, Formlabs introduced Form 2 as an accurate but affordable SLA-based 3D printer. Form 2 was one of the first 3D printers to be used in the dental office. ${ }^{9,10}$ While the concept of CAD/CAM crown fabrication was introduced in 1971, it was not until the last two decades that intraoral scanning technology, design software, and computational processes have popularized the in-office CAD/CAM restorative process (Figure 1).

\section{Brief History of Digital Dentistry}

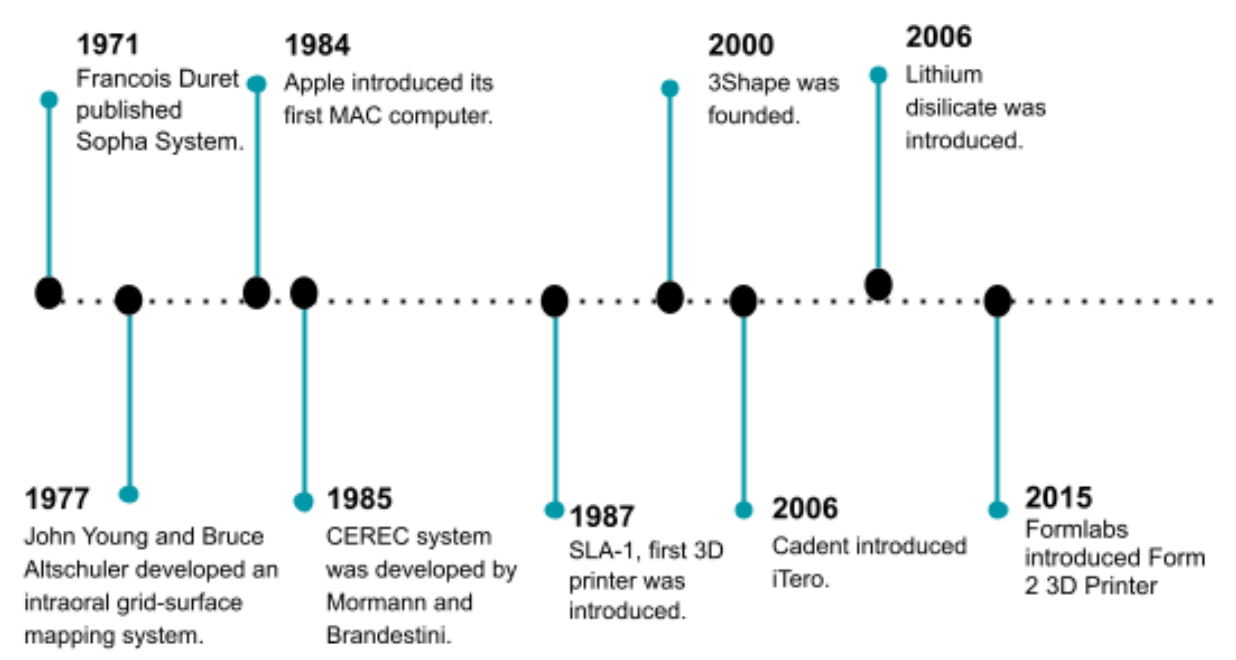

Figure 1. Brief History of CAD/CAM Dentistry includes timeline and inventions.

\subsection{Common Workflow for Same-day Dentistry}

The common digital workflow for same-day dentistry consists of abutment preparation (natural teeth or implant abutments), intraoral scanning, restoration design via a software suite, milling of the chosen restorative material by a milling system, postmilling sintering and characterization, and lastly, the luting/cementation process. This review describes the same-day dentistry process, from the basic abutment preparation through cementation of the restoration, with an emphasis on new technologies and advancements.

\section{Materials and Methods}

\subsection{Indications and Contraindications for Same-day Dentistry}

Over the past two decades, CAD/CAM dentistry has evolved to allow same-day preparation, fabrication, and delivery of multiple single-units of fixed prosthodontics. The indications and contraindications for use have also evolved based upon the advancements of available technologies (Table 1). 
Table 1. Indications and Contraindications for Same-day Dentistry

\begin{tabular}{ll}
\hline \multicolumn{1}{c}{ Indications } & \multicolumn{1}{c}{ Contraindications } \\
\hline $\begin{array}{l}\text { Ideal crown preparation } \\
\text { Angulation of the preparation is optimal for intraoral }\end{array}$ & Subgingival margin \\
scanning & Angulated abutment not allowing intraoral scanning \\
$\begin{array}{l}\text { Optimal mouth opening } \\
\text { Esthetics (optimal for monolithic or minor layering } \\
\text { ceramics) }\end{array}$ & Limited mouth opening \\
Single unit restorations & Esthetics (may require complex layered ceramics) \\
\hline & Fixed partial dentures or multiple single units \\
\hline
\end{tabular}

\subsection{Current Concepts on Abutment Preparation and Finishing Line}

$\mathrm{CAD} / \mathrm{CAM}$ abutment preparation is similar to conventional abutment preparation recommendations for all-ceramic restorations, taking into consideration the allowable minimal thickness of selected ceramic materials. For example, it is generally accepted that a $1 \mathrm{~mm}$ minimum thickness overall is appropriate for lithium disilicate. However, in areas of higher occlusal loads, or an area that may require customized layering of ceramic materials over the monolithic lithium disilicate material, greater tooth preparation may be required to allow for $1.5 \mathrm{~mm}$ to $2.0 \mathrm{~mm}$ thickness of the final material. ${ }^{11}$ Depending on the yttria concentration of zirconia, the minimum thickness of $3 \mathrm{MOL} \%$ yttria monolithic zirconia material can be as low as $0.7 \mathrm{~mm}$ to $1 \mathrm{~mm}$ thickness for posterior restorations under heavy occlusal load. ${ }^{12}$ While the concept of abutment preparation has not changed greatly from conventional, non-digital abutment preparation, the abutment preparation associated with CAD/CAM dentistry must serve four unique purposes: providing optimal material thickness, creating an appropriate path of restorative insertion, allowing access for accurate intraoral scanning, and allowing optimal manufacturing of the restorations through milling or possibly printing.

The monolithic nature of CAD/CAM materials enables a minimal thickness, thus giving clinicians the opportunity to prepare natural tooth abutments in a more conservative way than ever. This includes the minimal preparation of the abutment finishing line margin. A recent study by Rizonaki ${ }^{13}$ evaluated three types of finishing line on a typodont system--rounded shoulder, chamfer, and feather-edge--in 30 lithium disilicate crowns, 10 for each type of finishing line. Microcomputer tomography was used to evaluate the internal fit, marginal gaps, and 3D cement volume. While all three finishing lines provided clinically acceptable values, the rounded shoulder preparation appeared to provide the smallest gap width and discrepancy in the $2 \mathrm{D}$ measurements. The internal gap width was smaller in the chamfer and feather-edge margins compared to that of the round shoulder. When measured in 3D, the cement gap was also smaller in the chamfer and feather-edge margins than the round shoulder margin. While all three finishing lines are clinically acceptable, the chamfer finish line design may provide a compromise between conservative tooth preparation and fitting of the restoration compared to the shoulder design, which requires more tooth preparation, and the feather-edge design, where it may be difficult to define the margin.

\subsection{Intraoral Scanner Technologies}

Companies do not usually disclose the technology behind their intraoral scanners, complicating research and education on intraoral scanners. More importantly, fast-paced changes in the intraoral scanning field often render even the latest published articles obsolete, due to the frequency of software and hardware updates. The three major technologies used in intraoral scanners are: 1) the triangulation principle, 2) active wavefront sampling, and 3) confocal technology. Each intraoral scanner applies one or more of these common technologies,sometimes together with other systemspecific technologies. ${ }^{14-16}$ The triangulation principle, as seen in the older version of CEREC scanners, such as CEREC AC bluecam (Dentsply Sirona, Charlotte, NC, USA), utilizes a projection of a multiple-light ray onto the object, such as abutment teeth. Each light ray is then reflected back to the sensor, and the distance between the projected ray and reflected ray can be measured. The known fixed angle between the projector and sensor allows the distance to the object to be calculated through Pythagoras' theorem. ${ }^{14}$ The active wavefront sampling principle, such as in the Lava COS intraoral scanner, constructs the three-dimensional object using images that are reflected from a series of lenses. The focus or out-of-focus principle allows distances to be calculated. Several intraoral scanners apply the confocal technology principle in combinations with other technologies in image acquisition and processing. The confocal technology-based intraoral scanners include iTero, TriOs, and Planmeca scanners (Figure 2). Confocal technology uses the confocal laser scanning principle, in which a laser beam is projected onto an object, and the reflected beam is 
received through a focal filter. Only the image in the focal point of the lens will be projected onto the sensor. Because the focal distance is known, the distance between the scanned object and the lens can be calculated. ${ }^{14}$

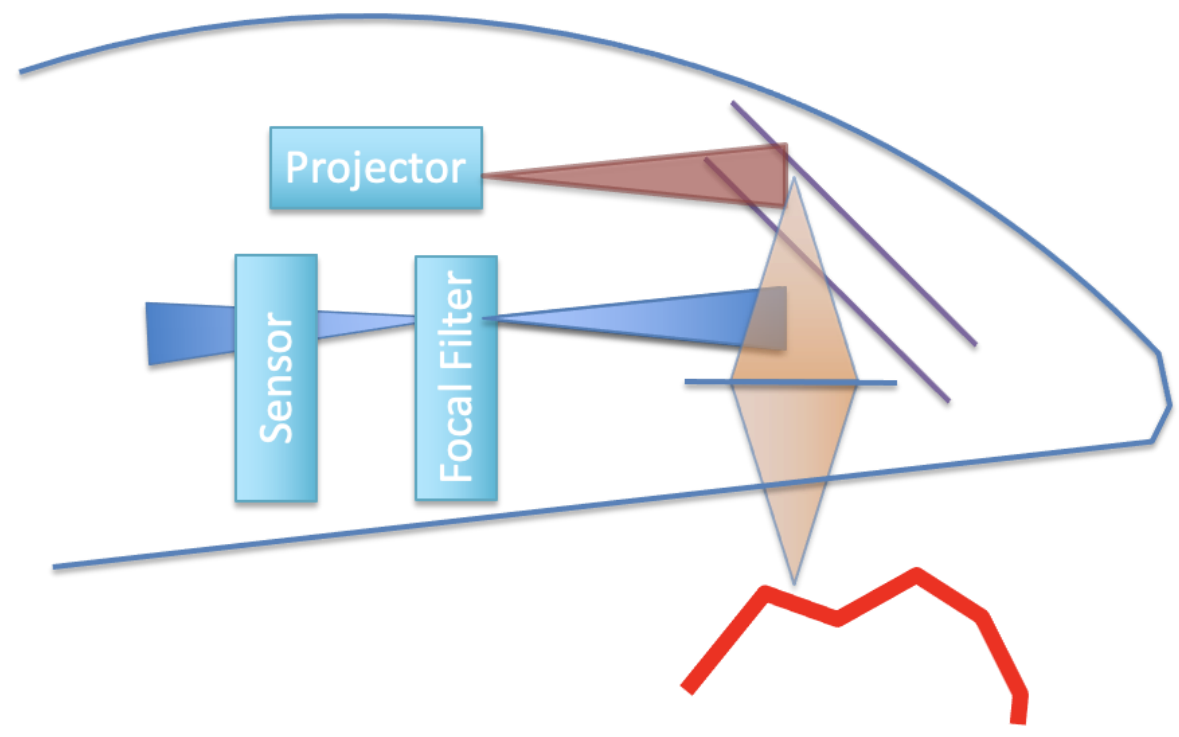

Figure 2. Basic components of the parallel confocal principle of an intraoral scanner are shown. The projector generates lasers that are reflected from mirrors and through the lens. The reflected lasers come back through the focal filter. Only the object in the focal point is received on the sensor.

\subsection{Accuracy of intraoral scanners}

The accuracy of intraoral scanners is often one of the most important comparative features for researchers and clinicians. Understanding the basic fundamentals of how these accuracy values are measured and the meaning of the values provides insight into clinical applications, subsequently improving patient care. Accuracy is often divided into two values: trueness and precision. Trueness represents the accuracy of measurement to the object that is measured or, in this case, scanned. Precision represents the consistency of the measurement. A highly accurate intraoral scanner should have high trueness and precision. In other words, an ideal intraoral scanner would produce a three-dimensional image that closely resembles the object being scanned. A high accuracy therefore allows the fabrication of dental prostheses through digital impressions. High trueness guarantees the fit of the prosthesis, while high precision allows software to compensate for known, common errors.

Accuracy of an intraoral scanner is dependent on multiple factors, including different proprietary hardware and technology, the number and distribution of abutments being scanned, full arch versus quadrant scanning, liquid contamination (heme or saliva) on the surface of the abutments, access and abutment angulations, ambient lighting, and rescanning mesh holes and stitching procedures. ${ }^{17}$ Zhang et al. ${ }^{18}$ examined the effects of number of abutments and abutment distributions on the accuracy of intraoral scanners. The more abutment teeth present, the more distortion of a scan, and the lower the accuracy value. The most distortion occurs in anterior and most posterior teeth. The anterior curvature produces more scanning distortion, and the distribution of the abutment teeth from the most posterior on each side of the arch can also contribute to more distortion.

Modern intraoral scanners produce an accurate scan in segmentation or quadrant area. A study by Ellakany ${ }^{19}$ compared three intraoral scanners with three extraoral desktop lab scanners. The results demonstrated that in an in vitro setting, the intraoral scanners performed just as well as the desktop scanners in segmentation or quadrant scannings. The difference was seen when scanning across the canine area. The curvature of the arch seemed to have an adverse effect on intraoral scanners compared to desktop scanners. 
Segmentation or quadrant scanning is therefore recommended whenever possible to accommodate current intraoral scanning technology, but it is possible that this will change in the future. Recent evidence suggests that room lighting (and possibly dental operatory lights) may have an adverse effect on the accuracy of an intraoral scanner. Clinicians should therefore check their operatory lights and/or turn them off during digital impression procedures. ${ }^{20}$ Because of the accuracy of modern intraoral scanners, it is now possible for clinicians to incorporate digital diagnostic design and 3D printing technology into their practice. This incorporation can enhance clinical techniques by enabling conservative tooth preparations through digital duplication and preparation guides. ${ }^{19,21,22}$

\section{Results}

\subsection{Staff Training and Team Development for CAD/CAM Same-day Dentistry}

McLaren and Culp ${ }^{23}$ pointed out the trend of incorporating emerging technology, including intraoral scanners and chair-side or in-house milling processes, which subsequently enhanced and popularized CAD/CAM same-day dentistry. They predicted that to support in-house, one-day restorations, a restorative dental practice would need new chair-side personnel, including a digital scanning technician/assistant, a digital prosthetic or restorative designer, and possibly an in-house ceramist.

While most practices and dental schools often quickly acquire hardware and software in order to support CAD/CAM dentistry through patient care as well as dental education, this approach has a flaw of neglecting personnel. Acquiring each new technology requires investment from the practice or the school, together with leadership and development of personnel, to support the transition, daily operations, as well as developing and prototyping appropriate workflow for a clinic or an institution.

Developing core staff members for CAD/CAM dentistry is fundamental to the long-term success of CAD/CAM dentistry in private practice and dental education.

The CAD/CAM dentistry education program at Virginia Commonwealth University (VCU) School of Dentistry demonstrated this principle. The educational components were integrated into each year of dental education. Dental students would learn how to use intraoral scanners at the end of their first year. Then, the students would do their scanning practice and learn software design during the fixed prosthodontic course in their second year. Then, in their third year, they come back to learn milling, sintering and characterization of restoration. In their third and fourth years in the clinic, they treat patients using digital technology in the CAD/CAM dentistry clinic. Over the course of 3 years, the clinic has increased CAD/CAM production from virtually 0 to over 1,000 single units (mostly same-day dentistry) and over 300 units of fixed partial dentures (FPD) using digital technology and the in-house laboratory (Figure 3). The students provided a high rating for their educational experiences during the annual survey (Figure 4). 


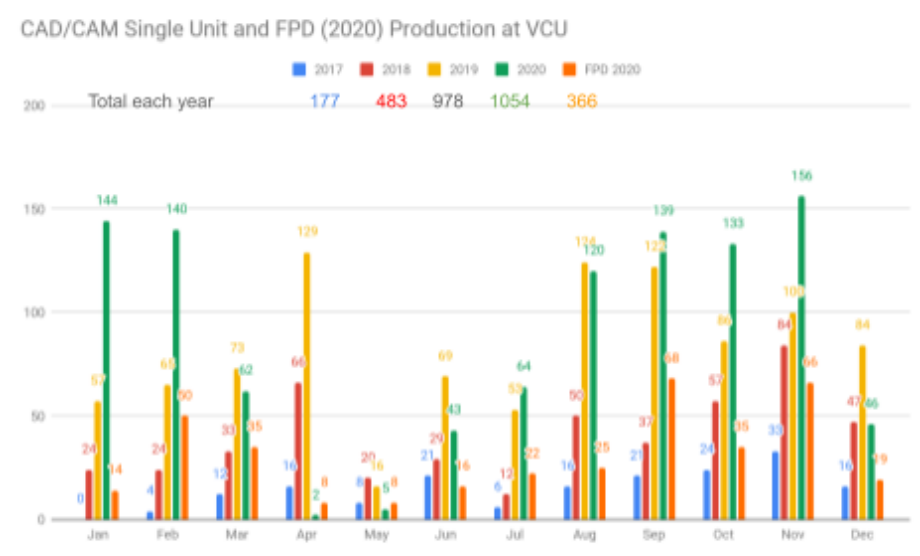

Figure 3. CAD/CAM Clinic Production demonstrates annual production in dental student clinics utilizing digital technology for fixed prosthodontics from 2017 to 2020.

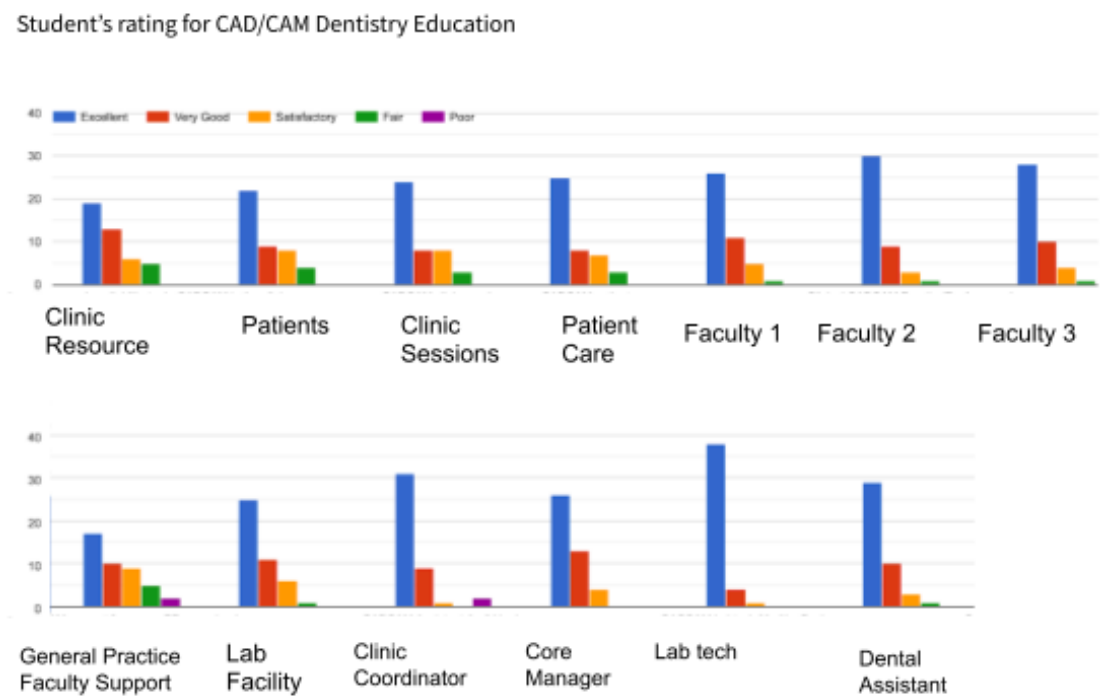

Figure 4. CAD/CAM Education Survey demonstrates students' level of satisfaction with the CAD/CAM education. The survey was an anonymous, annual quality improvement process. 45 out of 100 senior dental students responded to the survey.

\subsection{CAD/CAM Dentistry Technology Applications in Dental Education}

CAD/CAM digital technology has improved dental education at the preclinical level as well as the clinical level. At the preclinical level, many studies demonstrated that digital technology enables the visualization of preclinical training in dental anatomy and fixed prosthodontics.

Utilizing Planmeca E4D software to compare the student's crown preparation with the ideal (master) preparation, Renne et al. ${ }^{24}$ applied a digital software (Compare, Planmeca, Helsinki, Finland) to compare the student's crown preparation with the ideal (master) preparation. By visualizing their preparation superimposed onto the master preparation, a student's grading and self-evaluation can be done consistently and reliably. An actual numerical evaluation generated by the E4D Compare software frees up faculty time and eliminates subjective judgments. The computer-based evaluation 
was also found to be more precise than the hand-graded method. A similar study was conducted in Europe by Schlenz et al., ${ }^{25}$ who showed that by comparing student's preparation and master preparation using computer software, students saw the technology as beneficials in terms of handling, didactic benefit, and motivation. However, students in this study seemed to prefer a human instructor's feedback regarding tips and clinical pearls rather than the digital, numerical, computer-based feedback. Furthermore, the utilization of intraoral scanners in the fixed prosthodontic lab seems to assure students' confidence in utilizing technology in their future practice. More than $90 \%$ of the students indicated that they could imagine using an intraoral scanner in their clinic.

This technology is not limited to abutment preparation in preclinical prosthodontic courses. Chiang et al. ${ }^{26}$ utilized similar technology to help students' self-assessment in a dental anatomy wax-up exercise. In this study, thirty-seven students completed the waxup of the maxillary left central incisor and traditional self-assessment. Then, the students used CAD/CAM software (Compare, Planmeca, Helsinki, Finland) to superimpose the wax-up of the original master wax-up to illustrate under- and over-contoured areas. This method enabled visualization of the students' wax-up compared to the master waxup in three dimensions. Students then completed another self-assessment and an exit survey. The authors concluded that digital technology helps students learn visualization of tooth anatomy better, complementing faculty's feedback, and therefore enhances students' understanding of dental anatomy. A combination of traditional lab techniques and digital technology appears to be a new trend. Afshari et al ${ }^{27}$ compared the traditional pathway of custom implant abutment fabrication to the combination of traditional and digital pathways as well as to the pure digital pathway. The authors concluded that the trend in digital dentistry education is to combine and integrate digital dentistry into conventional workflow, and that incremental replacement of traditional workflow with digital workflow may provide benefits from both pathways, allowing students to develop their hand skills as well as enhance their understanding of prosthodontic principles.

\section{Discussion}

\subsection{Comparing Digital Workflow with Conventional Workflow}

Digital impressions and fabrication processes using intraoral scanners, digital design software, and in-house or in-lab milling systems have fundamentally changed the practice of restorative dentistry. Developing a customized workflow to match individual practices or institutions--including developing teamwork, choosing software and hardware, and establishing workflow--would depend on several factors. Clinicians or dental faculty/administrators need to consider the following factors. First, the practice or institution would prescribe a same-day dentistry protocol (in-house fabrication), or intraoral scanning only and outsource the prosthetic fabrication, or both. Second, the practice or institution would put an emphasis on the restorations of natural teeth, dental implants, or both. Third, the practice or institution would consider doing implant treatment planning using digital treatment planning--if so, the in-house or outsource design and fabrication of implant surgical guides for guided surgery should be considered. Fourth, the main production workflow would be selected for fixed prosthodontics only or both fixed and removable prosthodontics. Finally, other prostheses or appliances may be considered, such as occlusal splints, Invisalign, or sleep apnea appliances. These can either be scanned only or both scanned and fabricated in the office.

Gjelvold et al. ${ }^{28}$ compared the effectiveness of digital and conventional fixed prosthetic fabrication in 42 patients who needed up to six units of tooth-supported 
single crowns and/or fixed partial prostheses. The patients were randomly assigned to either digital or conventional impressions/workflows. The procedure times, dentists' and patients' assessments, and clinical evaluation of the restorations were assessed. Digital impressions used less time and were more favorable to both patients and dentists. In terms of restorative evaluation, while there were no statistical differences between the marginal integrity and interproximal contact of the restorations fabricated either by conventional or digital workflows, the digital fabricated ones had better occlusal contacts compared to the conventional ones.

Digital impressions appear to be more favorable to patients in routine restorative procedures supported by natural teeth. Similar results were seen with patients receiving dental implant restorations. A randomized, crossover-designed study by Joda and Bragger ${ }^{29}$ compared conventional and digital impressions for a single implant restoration. The authors concluded that patients favor digital impressions over conventional impressions. Bad taste of conventional impressions was the most common complaint. Digital impressions also seemed to be faster and more comfortable than conventional impressions. Digital impressions are therefore superior to conventional impressions in terms of patient's perception and experience, cost efficiency, environmental impact (by producing less discarded materials), reproducibility, and reduced transportation (by eliminating at least one trip to the lab or completely eliminating transportation through same-day dentistry).

Digital workflows, especially single units and fixed partial dentures, can be done without any physical dies or dental casts. Dental cast-less or model-less fabrication is often looked down upon by conventionally trained dentists. However, recent evidence suggests that dental cast-less fabrication may not only simplify lab processes, save time, and reduce costs, but the dental cast-less fabrication process also produces better fitting restorations. A randomized, controlled crossover-designed clinical trial by Joda et al. ${ }^{30,31}$ compared two digital workflows to a conventional workflow control with dental castless fabrication for implant-supported, fixed, partial dentures. Twenty patients received three monolithic zirconia prostheses based on all three modalities. Clinical time in the fitting and adjustment process during prosthetic insertion and cost of each workflow were compared. The digital process was clearly less expensive and faster to perform in the lab. However, one of the tested digital workflows was better in terms of insertion time saving and adjustment needed compared to the conventional control. The other digital workflow performed poorer than the conventional control. This suggests that optimization of digital workflow to individual practice and workflow specific technology may play an important role in the accuracy of the digital workflow, and in turn the quality of the fabricated prostheses. Understanding the digital workflow process - in particular, the intraoral scanning, software design, and milling process - is therefore crucial for optimal digital prosthesis fabrication (Figure 5).

Recent studies further suggest that regular software updates are important in maintaining the accuracy of intraoral scanners. It is also shown that older versions of scanning, and possibly designing, software can produce more error and likely more defective prostheses. ${ }^{32,33}$ 


\section{Design software}
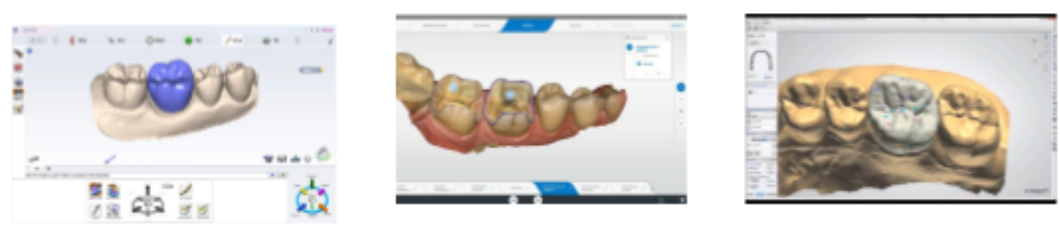
- Open system
- Link to IOS
- Allow manual manipulation of the restoration
- Simple design features
- Some automated
- Difficult to design multiple units at the same time but allow
duplication

Cerec

- Closed system

- Link to IOS

- More automated restorative design

- Restorative design based on adjacent teeth

- Difficult to design multiple units at the same time but allow duplication
3Shape

- Open system

- Not usually link to IOS

- Allow virtually unlimited design feature

- More difficult to master

- Capable of design multiple units at the same time.

- Also allow duplication

Figure 5. Features of Common Design Software for Same-day Restorations should be understood and optimized to fit individual clinical practice as well as regularly updated.

\subsection{Milling Systems: Milling Materials and Milling Machinery}

Most dental restorative, definitive, provisional, or intermediate materials can be milled using most current milling systems (Figure 6). Two of the most commonly used materials for same-day dentistry and in-house fabrication of fixed prostheses are lithium disilicate and zirconia. While lithium disilicate material has remained largely unchanged since its introduction in 2006, zirconia material has further developed and modified to optimize the material's physical and esthetic properties. The milling systems of these two materials are similar in both design and functionality; however, lithium disilicate milling is often accompanied by a water-based coolant to reduce frictional heat generated during the milling process. Unsintered lithium disilicate shrinks by only $\sim 0.2-$ $0.3 \%$ of its volume after sintering, ${ }^{34}$ as the unsintered material is relatively dense and therefore allows limited water penetration. In contrast, unsintered zirconia material has higher porosity, and it shrinks by $18-24 \%$ of its volume after sintering. ${ }^{35}$ The porosity of the unsintered zirconia makes it prone to water contamination, which can, in theory, adversely affect the physical properties during the sintering process. 


\section{Milling Machines}

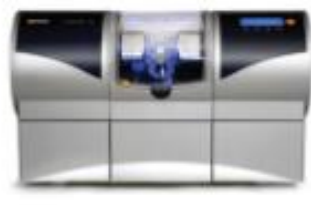

Cerec MCXL 4-axis

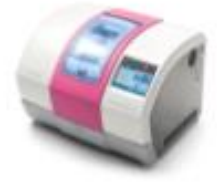

Planmeca PlanMill 30S

4-axis single spindles

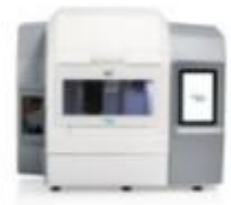

Ivoclar Vivadent

PrograMill PM7

5 -axis fully automated

Figure 6. Examples of current milling systems that are either 4- or 5-axis milling machines and capable of producing milling lithium disilicate, zirconia, or other materials. The Cerec and Planmeca milling machines are perhaps the most common same-day dentistry workflow capable of milling material in a block form. The Ivoclar Vivadent ProgramMill PM7 system is more suitable for lab-based milling and is capable of milling most millable dental materials.

\subsection{Sintering and Characterization of Definitive Restorations: Lithium Disilicate v.s. Zirconia}

The sintering process and time can be a significant factor in the workflow, especially for same-day restorations. Lithium disilicate often requires only a short period of time for sintering and potentially simultaneous characterization and glazing, takingabout 1015 minutes. Including time to cool down, the process takes about 30 to 45 minutes. With this in mind, a lithium disilicate restoration can easily be completed in one half-day, including tooth preparation, intraoral scanning, restorative designing and milling, optional try-in the unsintered stage, final trying of the definitive restoration, and cementation. Zirconia, on the other hand, requires a longer sintering time, especially the more translucent zirconia with a higher yttria content. Chairside "fast-sintering" CAD/CAM zirconia blocks (e.g., 3M Chairside zirconia, 3M and Katana STML) have been introduced to reduce sintering time from 8 hours to 20 minutes by using special speed-sintering furnaces (e.g., CEREC Speedfire, Dentsply). ${ }^{36}$ Post-sintering, zirconia also requires polishing and glazing. Understanding the materials used in practice will help you choose an appropriate milling machine and sintering furnace (Figure 7). 


\section{Ceramic Furnaces}

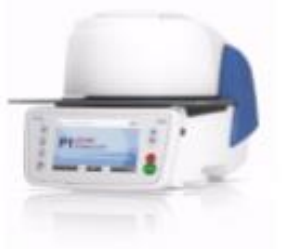

Programat CS3

Lithium disilicate Leucite
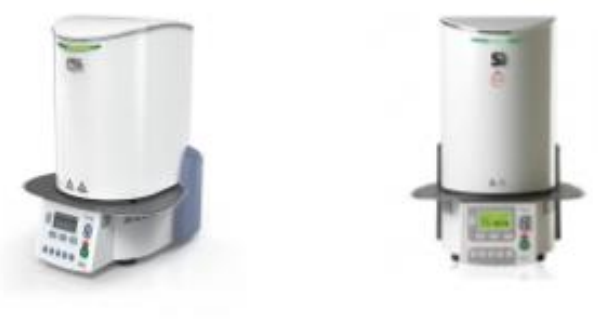

Programat CS4 Zirconia (fast sintering)
Lithium disilicate

Leucite

Figure 7. Examples of current sintering and glazing furnace systems: each system is designed for sintering and glazing of specific materials.

\subsection{Luting Cements and Cementation Procedures}

Monolithic CAD/CAM ceramic restorations can be luted with conventional cements (such as resin modified glass ionomer) or with composite-based resin cements, depending on the preparation design of the abutment. ${ }^{37}$ However, bonding of lithium disilicate and zirconia has recently been shown to provide additional retention and possibly a longer-lasting survival of the restoration. For lithium disilicate, a $5 \%$ hydrofluoric acid and silane are often used. A more recent innovation from Ivoclar Vivadent (Monobond Etch and Prime) combines both the etchant and the primer in one application (Figure 8). The conditioner appeared to roughen the surface of lithium disilicate, seen in electron microscopy to have a similar effect to the hydrofluoric acid, and produced a cohesive failure during debonding testing. ${ }^{38,39}$ However, while the bond strength may be adequate for restorations that do not require additional retention, it is still lower than that of hydrofluoric acid and silane. As a result, restorations that require maximum retention form should continue to use hydrofluoric acid and silane applications.

While lithium disilicate bonding has been performed successfully over the past 15 years, zirconia bonding is more controversial and not universally accepted. Blatz et al. ${ }^{40}$ suggested the following protocol for zirconia bonding, including A: Air particle abraded with alumina oxide $(50 \mu \mathrm{m}$ particle size at a pressure of $2.8 \mathrm{bar}$ for $12 \mathrm{~s}$ from a distance of $10 \mathrm{~mm}$ ), P: 10-Methacryloyloxydecyl dihydrogen phosphate (MDP) and C: Composite luting agent. This APC protocol has since been adopted as a standard for zirconia bonding. The majority of ceramic primers have included MDP in their formula, which can promote higher chemical bonds to zirconia. ${ }^{40}$ One of the issues of zirconia bonding is that zirconia has a high affinity for protein molecules in saliva. Saliva contamination is therefore a significant issue in zirconia bonding. A recent study by Sulaiman et al. ${ }^{41}$ compared non-contaminated zirconia samples with various methods of saliva decontaminations. They found that air-borne particle abrasion after saliva contamination is perhaps the most effective method to decontaminate saliva from the zirconia surface providing the highest bond strength. Alternatively, solutions designated as 'zirconia cleaners' can also be used to clean the zirconia surface after saliva contamination. 


\section{Basic Components of Monobond Etch \& Prime}

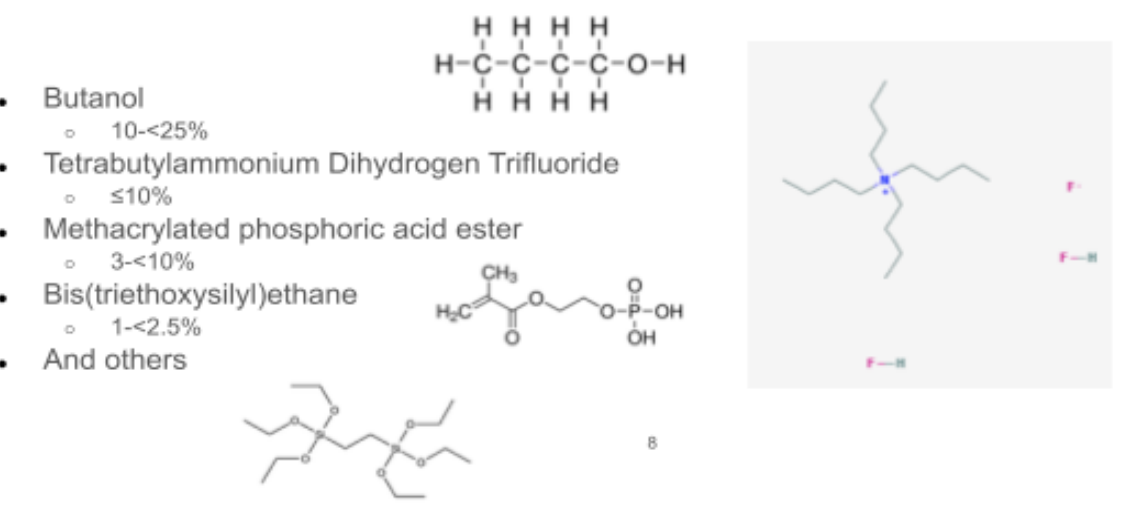

Figure 8 Basic Components of Monobond Etch \& Prime demonstrates each component in estimation: butanol (solvent), tetrabutylammonium dihydrogen trifluoride (etching agent), methacrylated phosphoric acid ester (chemical bonding reagent for the resin cement), and Bis(triethoxysilyl)ethane (silane agent).

\subsection{Case Study}

The process of same-day dentistry restoration is often referred to as the fabrication of a crown in one dental visit (Figure 8). The patient usually requires only a few single-unit fixed prosthodontics. Preoperative scans and diagnostic wax-up may sometimes be helpful, but in most cases, the existing tooth may be sufficient. The following case study demonstrates a patient with a maxillary left lateral incisor that required a crown restoration after a history of trauma and endodontic therapy. Preoperative scanning was performed, and the patient's existing tooth was used as a template for the definitive crown (Figure 9A). A conservative abutment preparation was performed with the facial margin at the gingival margin (Figure 9B). The abutment was then scanned and designed (Emerald, Planmeca, Helsinki, Finland). The lithium disilicate material (IPS, e.max CAD, Ivoclar Vivadent, Liechtenstein) was milled and tried in (Figure 9C). The crown was sintered, characterized, and glazed per the manufacturer's instructions. The definitive crown was then bonded to the abutment using a conditioner to the crown (Monobond Etch \& Primer, Ivoclar Vivadent) and phosphoric acid etching (Figure 9D). The crown was luted with dual-polymerizing cement (Variolink Esthetic DC, Ivoclar Vivadent, Liechtenstein). This case of same-day dentistry protocol was performed in the predoctoral clinic at the VCU CAD/CAM clinic (Figure 9E). 

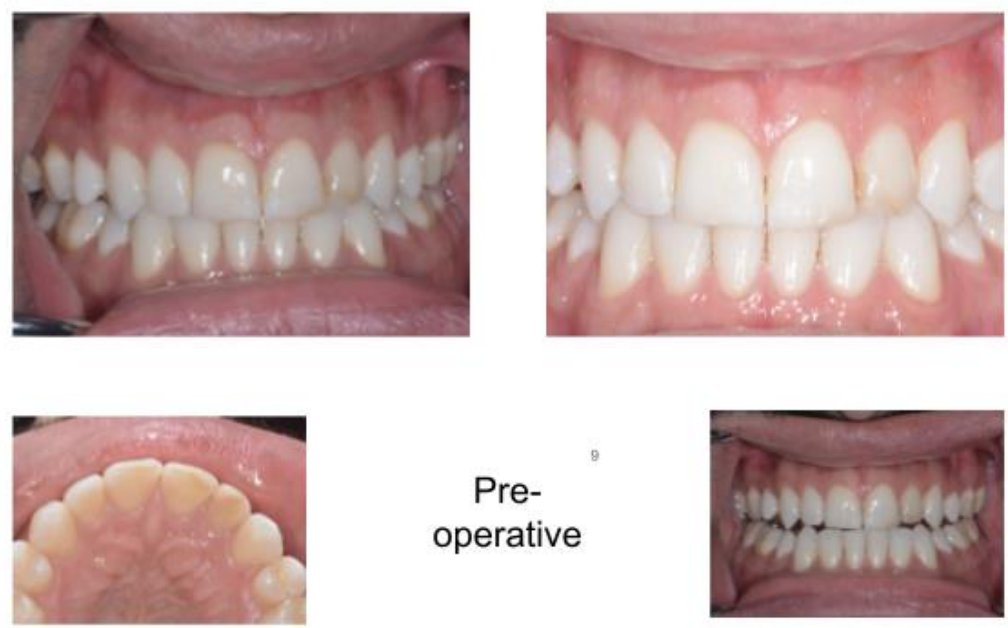

Pre-

operative

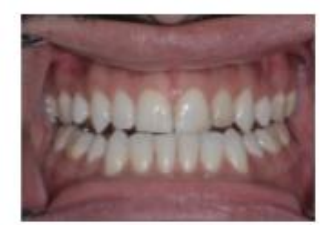

A
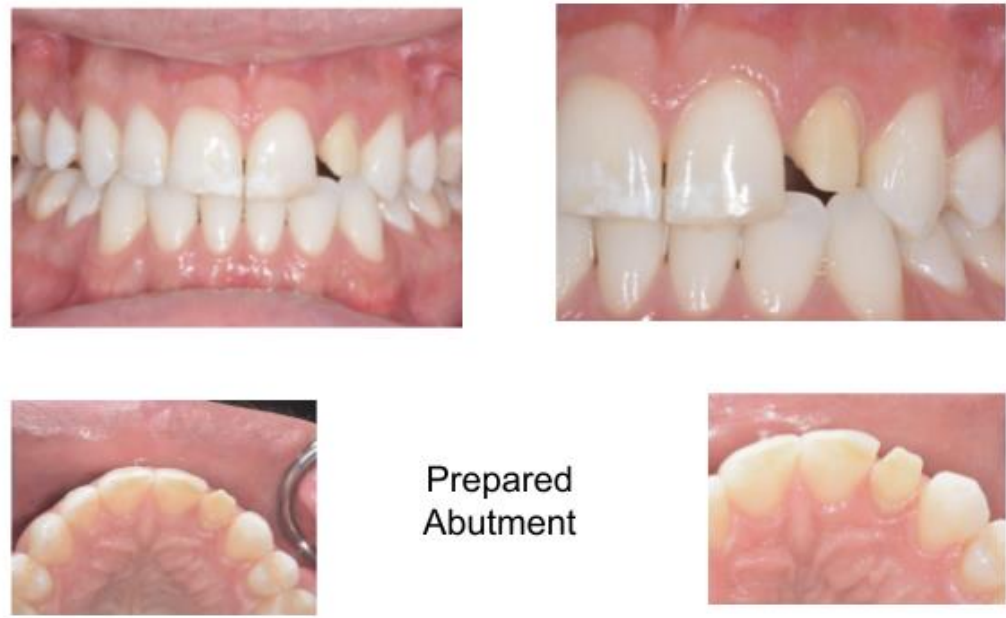

Prepared

Abutment

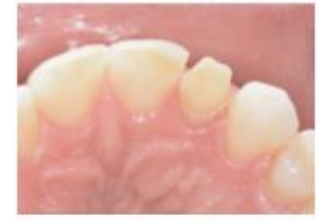

B
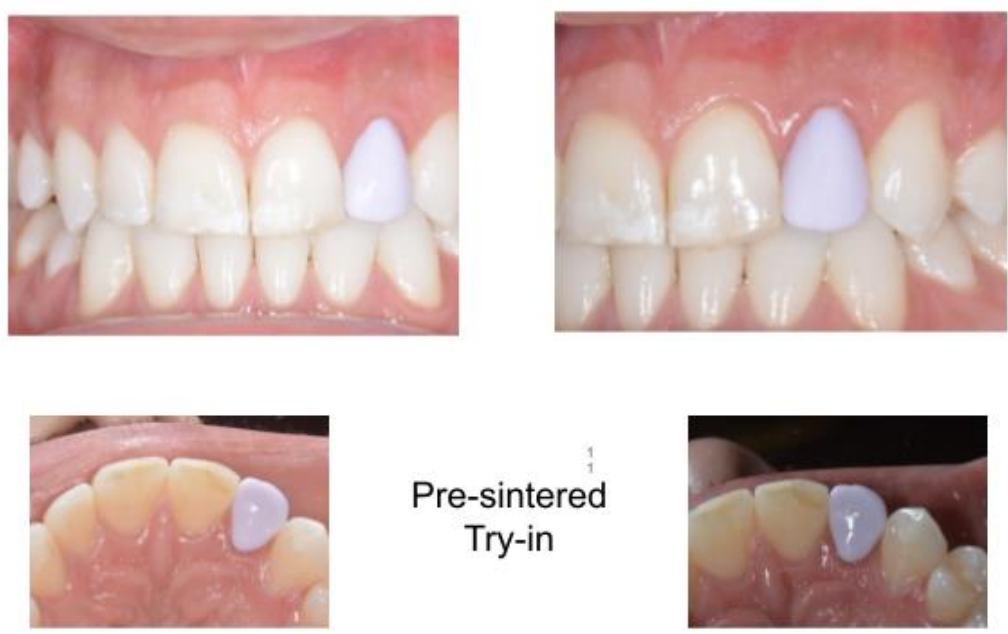

Pre-sintered

Try-in

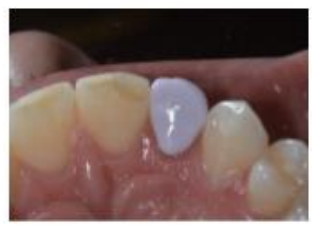

C 

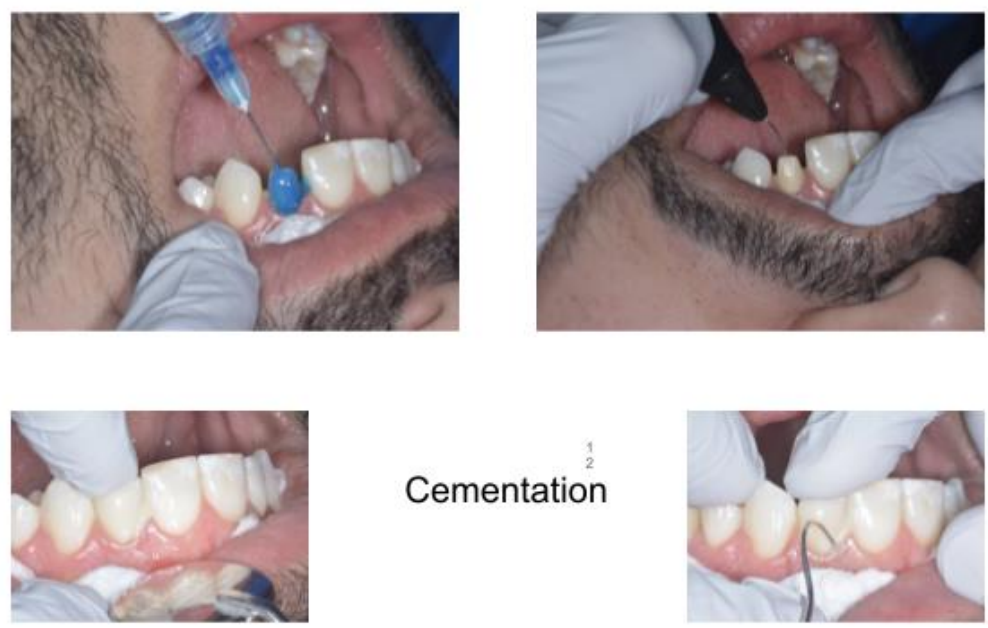

Cementation

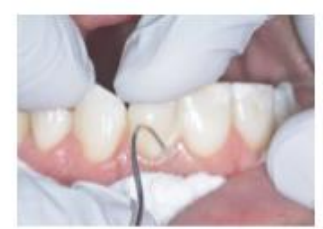

D
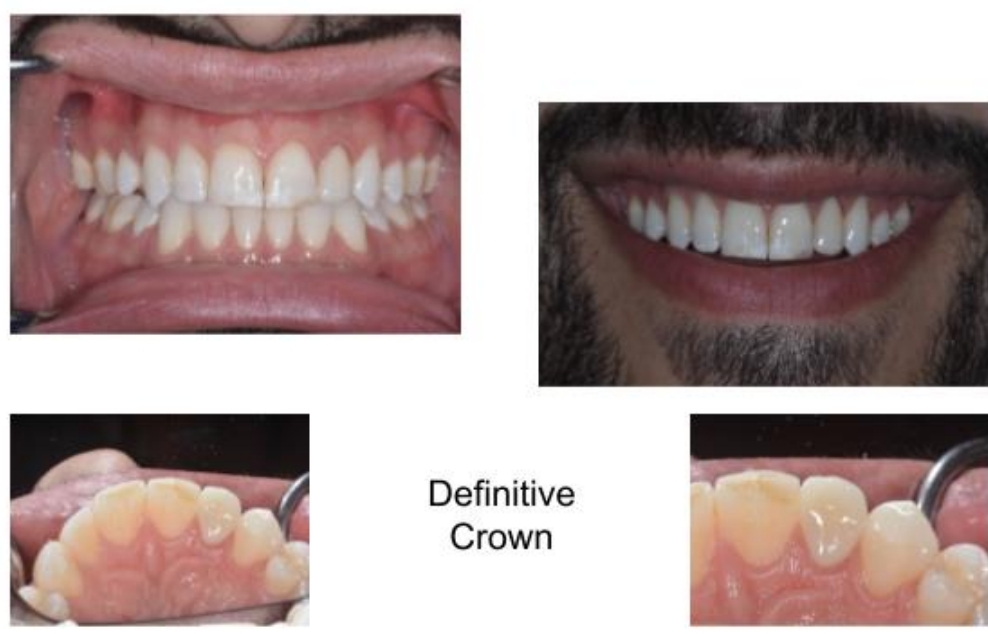

Definitive

Crown

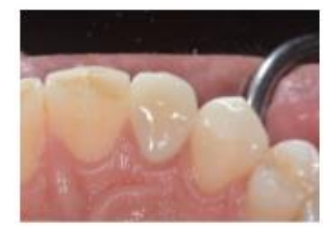

E

Figure 9 Clinical Steps for a Same-Day Crown* demonstrates a step-by-step from preoperative stage (A), crown preparation (B), Unsintered stage try-in (C), Bonding and luting process (D), and postoperative crown in place (E).

*A written consent form was obtained from the patient.

\section{Conclusions}

Same-day CAD/CAM dentistry has evolved as a result of advancement in intraoral scanning, restorative software design, mill processes, as well as new dental personnel and expertise. Same-day dentistry leverages advanced technologies together with new materials and clinical workflow. Clinicians must update their clinical practice and train their staff to develop their own customized digital workflow for their institution or practice. Digital dentistry is a disruptive technology that will continue to evolve and ultimately change dentistry to improve patient care and outcomes. 
Author Contributions: “Conceptualization, S.B; resources, S.B., L.O.S.; writing-original draft preparation, S.B., G.C.; writing - review and editing, S.B., T.A.S., L.O.S., W.A.C.; visualization, S.B.; All authors have read and agreed to the published version of the manuscript.

Funding: This review received no external funding.

Acknowledgments: The authors thank the faculty and staff at the Virginia Commonwealth University School of Dentistry Center of Digital Dentistry, in particular Marithe Baclagon, April Harris, Robert Armstead, Dr. Rami Ammoun, and Dr. Awab Abdulmajeed. Thanks also to current dental students Coleman Adams and Tam Nguyen, and former dental students Dr. Matthew Yeung, Dr. Nishal Dalal, Dr. Robert Rendenz, Dr. Cuong To, Dr. Henrique Silva, Dr. Daniel Baker, and Dr. Ali Ashkanani for their contributions and clinical work shown in this review. The authors also thank the faculty and staff associated with the University of North Carolina at Chapel Hill Center for Digital Innovation at the Adams School of Dentistry.

Conflicts of Interest: The authors declare no conflict of interest.

\section{References}

1. Duret, F., Blouin, J. L. \& Duret, B. CAD-CAM in dentistry. J. Am. Dent. Assoc. 117, 715-720 (1988).

2. Young, J. M. \& Altschuler, B. R. Laser holography in dentistry. The Journal of Prosthetic Dentistry vol. 38 216-225 (1977).

3. Williams, G. Apple Macintosh computer. Byte 9, 30-31 (1984).

4. Mörmann, W., Brandestini, M., Ferru, A., Lutz, F. \& Krejci, I. Marginal adaptation of adhesive porcelain inlays in vitro. Schweiz. Monatsschr. Zahnmed. 95, 1118-1129 (1985).

5. Mörmann, W. H. The evolution of the CEREC system. J. Am. Dent. Assoc. 137 Suppl, 7S-13S (2006).

6. Hull, C. W. Apparatus for production of three-dimensional objects by stereolithography. United States Patent, Appl., No. 638905, Filed (1984).

7. About 3Shape - We're on a Mission to Change Dentistry. https://www.3shape.com/en/our-story.

8. Jones, P. The iTero optical scanner for use with Invisalign: A descriptive review. Dent. Implantol. Update 19, 1-4 (2008).

9. Whitley, D. \& Bencharit, S. Digital implantology with desktop 3D printing. eacpds.com.

10. Whitley, D., 3rd, Eidson, R. S., Rudek, I. \& Bencharit, S. In-office fabrication of dental implant surgical guides using desktop stereolithographic printing and implant treatment planning software: A clinical report. J. Prosthet. Dent. 118, 256-263 (2017).

11. Fabbri, G. et al. Clinical evaluation of 860 anterior and posterior lithium disilicate restorations: retrospective study with a mean follow-up of 3 years and a maximum observational period of 6 years. Int. J. Periodontics Restorative Dent. 34, (2014).

12. Abdulmajeed, A., Sulaiman, T., Abdulmajeed, A., Bencharit, S. \& Närhi, T. Fracture load of different Zirconia types: A mastication simulation study. J. Prosthodont. 29, 787-791 (2020).

13. Rizonaki, M. et al. Evaluation of marginal and internal fit of lithium disilicate CAD-CAM crowns with different finish lines by using a micro-CT technique. J. Prosthet. Dent. (2021) doi:10.1016/j.prosdent.2020.11.027.

14. van der Meer, W. J., Andriessen, F. S., Wismeijer, D. \& Ren, Y. Application of intra-oral dental scanners in the digital workflow of implantology. PLoS One 7, e43312 (2012).

15. Logozzo, S., Zanetti, E. M., Franceschini, G., Kilpelä, A. \& Mäkynen, A. Recent advances in dental optics - Part I: 3D intraoral scanners for restorative dentistry. Optics and Lasers in Engineering vol. 54 203-221 (2014).

16. Logozzo, S., Kilpelä, A., Mäkynen, A., Zanetti, E. M. \& Franceschini, G. Recent advances in dental optics - Part II: Experimental tests for a new intraoral scanner. Optics and Lasers in Engineering vol. 54 187-196 (2014).

17. Kihara, H. et al. Accuracy and practicality of intraoral scanner in dentistry: A literature review. Journal of Prosthodontic Research vol. 64 109-113 (2020).

18. Zhang, T. et al. Evaluating the accuracy of three intraoral scanners using models containing different numbers of crownprepared abutments. Journal of Dental Sciences (2021) doi:10.1016/j.jds.2021.04.017.

19. Ellakany, P., El Tantawi, M., Mahrous, A. A. \& Al-Harbi, F. Evaluation of the Accuracy of Digital Impressions Obtained from Intraoral and Extraoral Dental Scanners with Different CAD/CAM Scanning Technologies: An In Vitro Study. Journal of Prosthodontics (2021) doi:10.1111/jopr.13400.

20. Revilla-León, M., Subramanian, S. G., Att, W. \& Krishnamurthy, V. R. Analysis of Different Illuminance of the Room Lighting Condition on the Accuracy (Trueness and Precision) of An Intraoral Scanner. Journal of Prosthodontics vol. 30 157-162 (2021).

21. Valenti, M., Schmitz, J. H., Cortellini, D., Valenti, A. \& Canale, A. A diagnostically and digitally driven tooth preparation protocol by using a patient monitoring tool with an intraoral scanner. The Journal of Prosthetic Dentistry (2021) doi:10.1016/j.prosdent.2021.04.017.

22. Valenti, M. \& Schmitz, J. H. A reverse digital workflow by using an interim restoration scan and patient-specific motion with an intraoral scanner. The Journal of Prosthetic Dentistry vol. 126 19-23 (2021).

23. McLaren, E. A., Culp, L. \& White, S. The evolution of digital dentistry and the digital dental team. Dent. Today 27, 112, 114, 116-7 (2008). 
24. Renne, W. G. et al. E4D compare software: an alternative to faculty grading in dental education. J. Dent. Educ. 77, 168-175 (2013).

25. Schlenz, M. A. et al. Undergraduate dental students' perspective on the implementation of digital dentistry in the preclinical curriculum: a questionnaire survey. BMC Oral Health 20, 78 (2020).

26. Chiang, H., Staffen, A., A Abdulmajeed, A., Janus, C. \& Bencharit, S. Effectiveness of CAD/CAM technology: A self-assessment tool for preclinical waxing exercise. Eur. J. Dent. Educ. 25, 50-55 (2021).

27. Afshari, F. S. et al. Integration of Digital Dentistry into a Predoctoral Implant Program: Program Description, Rationale, and Utilization Trends. J. Dent. Educ. 81, 986-994 (2017).

28. Gjelvold, B., Chrcanovic, B. R., Korduner, E.-K., Collin-Bagewitz, I. \& Kisch, J. Intraoral Digital Impression Technique Compared to Conventional Impression Technique. A Randomized Clinical Trial. J. Prosthodont. 25, $282-287$ (2016).

29. Joda, T. \& Brägger, U. Patient-centered outcomes comparing digital and conventional implant impression procedures: a randomized crossover trial. Clin. Oral Implants Res. 27, e185-e189 (2016).

30. Joda, T. et al. Time-efficiency and cost-analysis comparing three digital workflows for treatment with monolithic zirconia implant fixed dental prostheses: A double-blinded RCT. J. Dent. 103779 (2021).

31. Gintaute, A. et al. A Double-Blind Crossover RCT Analyzing Technical and Clinical Performance of Monolithic ZrO2 Implant Fixed Dental Prostheses (iFDP) in Three Different Digital Workflows. Journal of Clinical Medicine vol. 102661 (2021).

32. Haddadi, Y., Bahrami, G. \& Isidor, F. Effect of Software Version on the Accuracy of an Intraoral Scanning Device. Int. J. Prosthodont. 31, 375-376 (2018).

33. Vág, J. et al. The effect of software updates on the trueness and precision of intraoral scanners. Quintessence Int. 52, 636-644 (2021).

34. Wang, H., Liao, Y., Chao, Y. \& Liang, X. Shrinkage and strength characterization of an alumina-glass interpenetrating phase composite for dental use. Dental Materials vol. 23 1108-1113 (2007).

35. Oh, G.-J., Yun, K.-D., Lee, K.-M., Lim, H.-P. \& Park, S.-W. Sintering behavior and mechanical properties of zirconia compacts fabricated by uniaxial press forming. J. Adv. Prosthodont. 2, 81-87 (2010).

36. Sulaiman, T. A. Materials in digital dentistry-A review. J. Esthet. Restor. Dent. 32, 171-181 (2020).

37. Zarone, F., Di Mauro, M. I., Ausiello, P., Ruggiero, G. \& Sorrentino, R. Current status on lithium disilicate and zirconia: a narrative review. BMC Oral Health 19, 134 (2019).

38. El-Damanhoury, H. M. \& Gaintantzopoulou, M. D. Self-etching ceramic primer versus hydrofluoric acid etching: Etching efficacy and bonding performance. J. Prosthodont. Res. 62, 75-83 (2018).

39. Scherer, M. M. et al. Fatigue failure load of an adhesively-cemented lithium disilicate glass-ceramic: Conventional ceramic etching vs etch \& prime one-step primer. Dent. Mater. 34, 1134-1143 (2018).

40. Blatz, M. B., Chiche, G., Holst, S. \& Sadan, A. Influence of surface treatment and simulated aging on bond strengths of luting agents to zirconia. Quintessence Int. 38, 745-753 (2007).

41. Sulaiman, T. A., Altak, A., Abdulmajeed, A., Rodgers, B. \& Lawson, N. Cleaning Zirconia Surface Prior To Bonding: A Comparative Study of Different Methods and Solutions. J. Prosthodont. (2021) doi:10.1111/jopr.13389. 